\title{
Cosmic-Ray Positrons Strongly Constrain Leptophilic Dark Matter
}

\author{
Isabelle John ${ }^{a, *}$ and Tim Linden ${ }^{a}$ \\ ${ }^{a}$ Stockholm University and The Oskar Klein Centre for Cosmoparticle Physics, \\ Alba Nova, 10691 Stockholm, Sweden \\ E-mail: isabelle.john@fysik.su.se, linden@fysik.su.se
}

Cosmic-ray positrons have long been considered a powerful probe of dark matter annihilation. In particular, myriad studies of the unexpected rise in the positron fraction have debated its dark matter or pulsar origins. In this work, we instead examine the potential for extremely precise positron measurements by AMS-02 to probe hard leptophilic dark matter candidates that do not have spectral features similar to the bulk of the observed positron excess. Utilizing a detailed cosmic-ray propagation model that includes a primary positron flux generated by Galactic pulsars in addition to a secondary component constrained by Helium and proton measurements, we produce a robust fit to the local positron flux and spectrum. We find no evidence for a spectral bump correlated with leptophilic dark matter, and set strong constraints on the dark matter annihilation cross-section that fall below the thermal annihilation cross-section for dark matter masses below $60 \mathrm{GeV}$ and $380 \mathrm{GeV}$ for annihilation into $\tau^{+} \tau^{-}$and $e^{+} e^{-}$, respectively, in our default model.

$37^{\text {th }}$ International Cosmic Ray Conference (ICRC 2021)

July 12 th - 23rd, 2021

Online - Berlin, Germany

\footnotetext{
*Presenter
} 


\section{Introduction}

The AMS-02 experiment provides extremely precise measurements of the flux, composition and spectrum of the local cosmic-rays [1]. These observations yield new insights into the energetics of the Galaxy's most powerful sources, and the properties of Galactic transport. Additionally, searches for unexpected spectral features can potentially provide evidence for new physics such as dark matter annihilation.

The AMS-02 experiment and earlier instruments have shown an unexpected rise in the positron flux above $20 \mathrm{GeV}$.Its dark matter or pulsar origin has been debated and recently the pulsar explanation has become favored (e.g. [2-6]). In this work, we attribute the rising positron flux to pulsars, and instead make use of the high-statistical precision of the AMS02 data to calculate strong constraints on dark matter annihilation into highly-peaked leptonic final states, which have spectra that are inconsistent with the overall structure of the rising positron fraction. A schematic example of such a positron spectrum is shown in Figure 1: At low energies, the positron flux is dominated by secondary positrons produced by protons and a small amount of Helium nuclei, while at higher

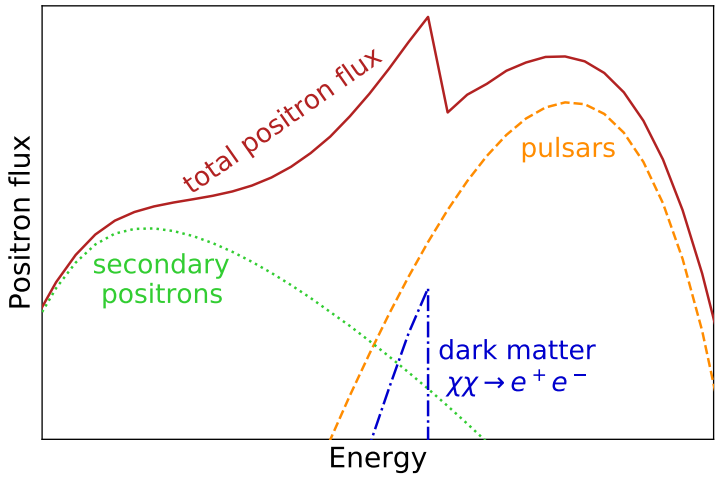

Figure 1: Sketch of the local cosmic-ray positron flux and its components as considered in this work. At low energies, secondary positrons are produced from protons and Helium. At higher eneriges, the flux is dominated by pulsar contributions. To this background we add sharply peaked contributions from dark matter that annihilates into leptonic final states. energies, most positrons are produced as $e^{+} e^{-}$pairs by pulsars. Against this background, our analysis probes a subdominant contribution from annihilating dark matter.

Our work builds on a similar analysis by Bergström et al. (2013) [7]. They examine dark matter models with masses between between $5-300 \mathrm{GeV}$ for annihilations into leptonic final states $\left(\tau^{+} \tau^{-}\right.$, $\mu^{+} \mu^{-}, e^{+} e^{-} \gamma$ and $e^{+} e^{-}$). Their strongest limits are obtained for the direct $e^{+} e^{-}$channel, where they set upper limits on the dark matter annihilation cross section of about $10^{-28} \mathrm{~cm}^{3} / \mathrm{s}$ at $5 \mathrm{GeV}$ and about $10^{-25} \mathrm{~cm}^{3} / \mathrm{s}$ at $250 \mathrm{GeV}$.

In our analysis, we update and improve on the Bergström et al. results in three ways: (1) Our analysis relies on a larger dataset with better statistical precision provided by the latest AMS-02 data [8-11]. (2) We use the cosmic-ray propagation code Galprop [12] to create a full cosmic-ray propagation model for the positron, proton and Helium. Our fit allows a large variety of parameters, such as the diffusion and particle injection spectra in the fit to float, while Bergström et al. build their background from a handful of pre-defined Galprop models. (3) We implement a new solar modulation model [13] that describes the effects on the cosmic-ray particles in the heliosphere more precisely, rather than approximating the solar modulation by a force-field potential.

The outline is as follows: In Section 2, we describe the methodology of this analysis and explain the data selection, the computational model, the dark matter model and the statistical analysis. The results are presented in Section 3, and our conclusions are discussed in Section 4. Further details, analysis checks, and discussions can be found in the companion paper [22]. 


\begin{tabular}{c|c|c|c}
\hline Particle & Energy range $[\mathrm{GeV}]$ & Time range & Ref. \\
\hline \hline Positrons & $2-1000$ & May 2011 - Nov. 2017 & {$[8]$} \\
\hline \multirow{2}{*}{ Protons } & $2-60$ & Feb. 2015 - May 2017 & {$[9]$} \\
& $60-1800$ & May 2011 - Nov. 2013 & {$[10]$} \\
\hline Helium & $2-1000$ & May 2011 - Nov. 2013 & {$[11]$} \\
\hline
\end{tabular}

Table 1: AMS-02 data sets used in this analysis. Since our solar modulation model is time-dependent, we are able to utilize datasets from different time periods.

\section{Methodology}

Our analysis generally proceeds as follows. To compute the dark matter limits, we perform a fit of the positron, proton and Helium data from AMS-02 to constrain the parameters of our astrophysical model in the absence of dark matter. Then we add a dark matter contributions to the local positron flux and compute the $95 \%$ confidence upper limit on the dark matter annihilation rate. Notably, we allow critical astrophysical parameters to be re-fit for each dark matter mass and cross-section, producing a more realistic constraint on the dark matter contribution. In the following sections, the procedure is described in more detail.

\subsection{Data Selection}

For our analysis, we use the recently published AMS-02 data for positrons, protons and Helium as summarized in Table 1. Since protons and Helium produce a large part of the positron flux, we include protons and Helium in the astrophysical model to obtain more realistic constraints on the model parameters, while the dark matter constraints only directly depend on the positron flux. For protons, we combine two different data sets - at lower energies, we select only data from after the polarity flip of the solar magnetic field to reduce uncertainties from the solar modulation.

\subsection{Computational Model}

We create our astrophysical background using the cosmic-ray propagation code Galprop, and tune the following Galprop parameters: the diffusion spectrum (diffusion coefficient $D_{0}$ at a reference rigidity of $4 \mathrm{GV}$, diffusion break $D_{\text {break }}$, and spectral indices $\delta_{1}$ and $\delta_{2}$ below and above the break), the proton injection spectrum (spectral break and indices $\gamma_{1}^{p}$ and $\gamma_{2}^{p}$ below and above the break), the Helium injection spectrum (spectral break and indices $\gamma_{1}^{\mathrm{He}}$ and $\gamma_{2}^{\mathrm{He}}$ below and above the break), the convection velocity $v_{c}$ and the Alfvén velocity $v_{\text {Alfvén }}$ (reacceleration). Noting that the dark matter contributions to cosmic-ray antiprotons depend sensitively on the halo height [25], we produce analyses for two different choices of the Galactic halo height. Here we show results for a halo height of $5.6 \mathrm{kpc}$, but in [22] we also show analyses for a conservative halo height of $3.0 \mathrm{kpc}$.

In our model, we introduce pulsars as a new source of primary positrons by modifying the Galprop code. For this, we adopt the profile for the pulsar distribution from [15], multiplied with the scale height given in [16] to get the 3-dimensional pulsar distribution in the Galaxy,

$$
\rho(R, z)=64.6 \mathrm{kpc}^{-4.35} R^{2.35} \exp \left[\frac{-R}{R_{0}}\right] \exp \left[\frac{-|z|}{z_{0}}\right],
$$




\begin{tabular}{l|c} 
Parameter & Best fit \\
\hline \hline Diffusion coefficient, $D_{0}\left[\mathrm{~cm}^{2} / \mathrm{s}\right]$ & $1.636 \cdot 10^{28}$ \\
Diffusion spectrum break, $D_{\text {break }}[\mathrm{MV}]$ & $6.067 \cdot 10^{3}$ \\
Spectral index below break, $\delta_{1}$ & 0.0527 \\
Spectral index above break, $\delta_{2}$ & 0.361 \\
Convection velocity, $v_{c}[\mathrm{~km} /(\mathrm{s} \mathrm{kpc})]$ & 6.345 \\
Alfvén velocity, $v_{\text {Alfvén }}[\mathrm{km} / \mathrm{s}]$ & 4.524 \\
Proton injection spectrum break [MV] & $5.195 \cdot 10^{2}$ \\
Proton spectral index below break, $\gamma_{1}^{p}$ & 1.657 \\
Proton spectral index above break, $\gamma_{2}^{p}$ & 2.523 \\
Pulsar spectral index, $\gamma_{\text {psr }}$ & 1.337 \\
Pulsar cutoff energy, $E_{\text {cut }}^{\text {psr }}[\mathrm{GeV}]$ & 535.587 \\
Pulsar formation rate, $\dot{N}_{100}[\mathrm{psr} / \mathrm{century}]$ & 0.0930 \\
Solar modulation parameter, $\phi_{0}[\mathrm{GV}]$ & 0.378 \\
Solar modulation parameter, $\phi_{1}[\mathrm{GV}]$ & 1.950 \\
Normalization (positrons, protons) & 0.815 \\
Helium injection spectrum break $[\mathrm{MV}]$ & $3.053 \cdot 10^{5}$ \\
Helium spectral index below break, $\gamma_{1}^{\mathrm{He}}$ & 2.505 \\
Helium spectral index above break, $\gamma_{2}^{\mathrm{He}}$ & 2.425 \\
Normalization (Helium) & 1.100 \\
\hline
\end{tabular}

Table 2: List of free parameters and their best-fit values for the astrophysical background model.

where $z$ is the halo height of the Galaxy, and $R_{0}=1.528 \mathrm{kpc}$ and $z_{0}=0.330 \mathrm{kpc}$. We take the pulsar $e^{+} e^{-}$spectrum from [2],

$$
\frac{d N}{d E} \approx C\left(\frac{E}{\mathrm{GeV}}\right)^{-\gamma_{\mathrm{psr}}} \exp \left[\frac{-E}{E_{\mathrm{cut}}^{\mathrm{psr}}}\right](\mathrm{GeVs})^{-1},
$$

where $\gamma_{\mathrm{psr}}$ is the spectral index and $E_{\mathrm{cut}}^{\mathrm{psr}}$ the cutoff energy. The normalization is given by $C=8.6 \cdot 10^{38} \dot{N}_{100}$, where $8.6 \cdot 10^{38}$ includes the moment of inertia and the period of the pulsar, and $\dot{N}_{100}$ is the pulsar formation rate in pulsars per century. In our model, we let $\gamma_{\mathrm{psr}}, E_{\mathrm{cut}}^{\mathrm{psr}}$ and $\dot{N}_{100}$ float.

Cosmic-rays are affected by the heliosphere of the Sun, causing particle losses at low energies ( $\lesssim 10 \mathrm{GeV}$ ). This effect also depends on the orientation of the solar magnetic field and solar activity. Often (e.g. as in [7]), the solar modulation is approximated by a force-field potential [14]. Here, we implement the solar modulation model from [13], which takes into account the time-, chargeand rigidity dependence of the solar modulation. We keep the parameters $\phi_{0}$ and $\phi_{1}$, that describe the heliospheric potential, as free parameters. All model parameters of the astrophysical model can be found in Table 2 .

\subsection{Dark Matter Model}

After obtaining the astrophysical model, we investigate the dark matter contribution in the positron flux. For the dark matter model, we assume a local dark matter density of $0.4 \mathrm{GeV} / \mathrm{cm}^{3}$ 

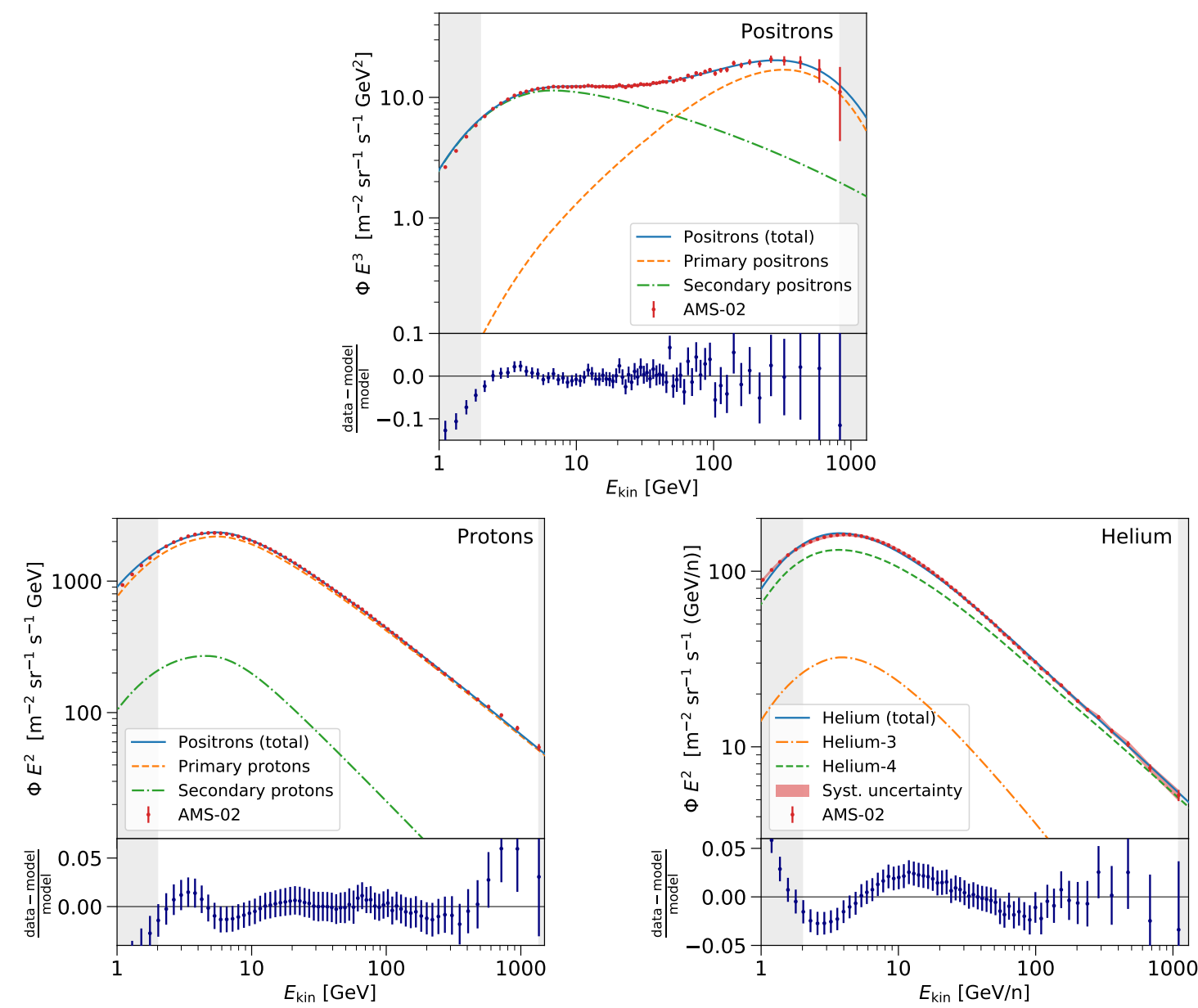

Figure 2: The astrophysical model for positrons (top), protons (bottom left), and Helium (bottom right), plotted in solid blue lines, together with the data from AMS-02 for positrons, protons and Helium, displayed in red. For positrons and protons, primary components are displayed in orange, secondary components in green. ${ }^{3} \mathrm{He}$ and ${ }^{4} \mathrm{He}$ are represented by orange and green, respectively. Note also that the Helium fit includes an additional systematic uncertainty band of $2 \%$. Gray shaded regions are excluded from the fit. The fractional residuals of the fits are at the bottom of each panel, showing that we fit all data to a few percent accuracy.

and a core radius of the dark matter halo of $20 \mathrm{kpc}$, and describe the dark matter distribution in the Galaxy by using an NFW profile [17]. We consider four different leptonic final states: $\chi \chi \rightarrow \tau^{+} \tau^{-}$, $\chi \chi \rightarrow \mu^{+} \mu^{-}, \chi \chi \rightarrow e^{+} e^{-}$and $\chi \chi \rightarrow \phi \phi \rightarrow e^{+} e^{-} e^{+} e^{-}$, where $\phi$ is a light mediator. For the $\tau^{+} \tau^{-}$, and $\mu^{+} \mu^{-}$final states, we use dark matter spectra obtained with DarkSUSY v.5.1.1 [18]. For the $e^{+} e^{-} e^{+} e^{-}$and $e^{+} e^{-}$states, we utilize analytic calculations of the dark matter spectrum that we directly implement in Galprop. Specifically, in the $e^{+} e^{-} e^{+} e^{-}$case, the contribution to the positron flux is constant for each energy bin and integrates to the dark matter mass for an energy range from approximately 0 to the dark matter mass, following a similar calculation as in Ref. [19]. The $e^{+} e^{-}$ final state is represented by a delta function.

To determine the dark matter limits, we study dark matter masses between 5 and $2000 \mathrm{GeV}$ for different annihilation cross sections $\langle\sigma v\rangle$, and select additional parameters that are particularly 
sensitive to, or are degenerate with, the dark matter contribution $\left(D_{0}, \delta_{2}, E_{\mathrm{cut}}^{\mathrm{psr}}, \gamma_{\mathrm{psr}}\right.$ and $\left.\dot{N}_{100}\right)$, and let them float as well when fitting to the positron data. This allows us to set more robust constraints.

\subsection{Statistical Analysis}

We fit our astrophysical background model to the AMS-02 data for positrons, protons and Helium simultaneously, using different minimizers (Multinest [20], iMinuit [21]) in two steps (for more information, see [22]). As we want to focus on the positron fit of our model, we introduce an additional systematic uncertainty of $2 \%$ in the Helium data. More importantly, the systematic uncertainties of nearby energy bins in the Helium flux are correlated, but here we treat them as uncorrelated, as the AMS-02 experiment does not provide the covariance matrices. The free parameters of our model are given in Table 2.

To obtain the dark matter limits, for each dark matter mass of the different final states, we create a grid of annihilation cross-sections and re-fit the positron flux using the relevant parameters (see above) for each grid point. Then we compute the $\chi^{2}$ profile of $\langle\sigma v\rangle$ and calculate the $95 \%$ upper confidence limit compared to no contribution from dark matter.

\section{Results}

The parameters of our astrophysical background model and their best-fit values are given in Table 2 . In general, our best-fit parameters are consistent with previous analyses [25, 27-29]. In Figure 2, we present our results of the astrophysical background model for the positron, proton and Helium flux. In the bottom of each panel, the residuals of our model are shown, indicating that our model fits the data to within a few percent. Table 3 summarizes the $\chi^{2}$

\begin{tabular}{c|c|c}
\hline Type & $\chi^{2}$ & $\chi^{2}$ /d.o.f. (d.o.f.) \\
\hline \hline total & 88.60 & $0.63(141)$ \\
positrons & 42.88 & $0.88(49)$ \\
protons & 21.08 & $0.43(49)$ \\
Helium & 24.64 & $0.57(43)$ \\
\hline
\end{tabular}

Table 3: Summary of the $\chi^{2}$-values of the fit of the astrophysical background model. values of the astrophysical background model. Due to the high-statistical precision of the data, which often falls below the systematic uncertainties in each flux, we are able to obtain $\chi^{2} /$ degree of freedom values below 1 in all cases.

In Figure 3, we present our dark matter limits on the annihilation cross section as a function of mass for the different final decay states, given as the $95 \%$ upper confidence limit. We derive the strongest constraints for dark matter annihilation directly into $e^{+} e^{-}$-pairs with an annihilation cross section of $6 \times 10^{-29} \mathrm{~cm}^{3} / \mathrm{s}$ for $12 \mathrm{GeV}$. For reference, we include the thermal cross section [24].

At energies below $10 \mathrm{GeV}$, our results show a small excess of $\lesssim 3 \sigma$ for all final states. In the positron fit in Figure 2, it can be seen that there is a positive discrepancy (about 2\%) between our model and the AMS-02 data below about $5 \mathrm{GeV}$, which causes the consistency with small dark matter contributions which produces a tentative preference for a small dark matter contribution. We note that this region of our model is prone to larger uncertainties, as it is close to the lower limit of our model $(2 \mathrm{GeV})$. Additionally, lower energies are highly affected by uncertainties in the solar modulation. 


\section{Discussion and Conclusion}

In this analysis, we calculated constraints on positron production from dark matter annihilation for dark matter masses from 5-2000 GeV for leptonic final states from the local positron flux. We created an astrophysical background model including a pulsar model to describe the rise in the positron flux above $20 \mathrm{GeV}$, and obtained very good agreement with the AMS-02 data. From this, we were able to derive strong constraints on the dark matter contribution to the positron flux, and exclude cross sections below the thermal cross section for annihilation into $\tau^{+} \tau^{-}$below $60 \mathrm{GeV}$, for $\mu^{+} \mu^{-}$below $160 \mathrm{GeV}$, for $e^{+} e^{-} e^{+} e^{-}$below $240 \mathrm{GeV}$ and for $e^{+} e^{-}$below $380 \mathrm{GeV}$. At lower masses near $30 \mathrm{GeV}$, we rule out dark matter annihilation to $e^{+} e^{-}$with cross-sections on the

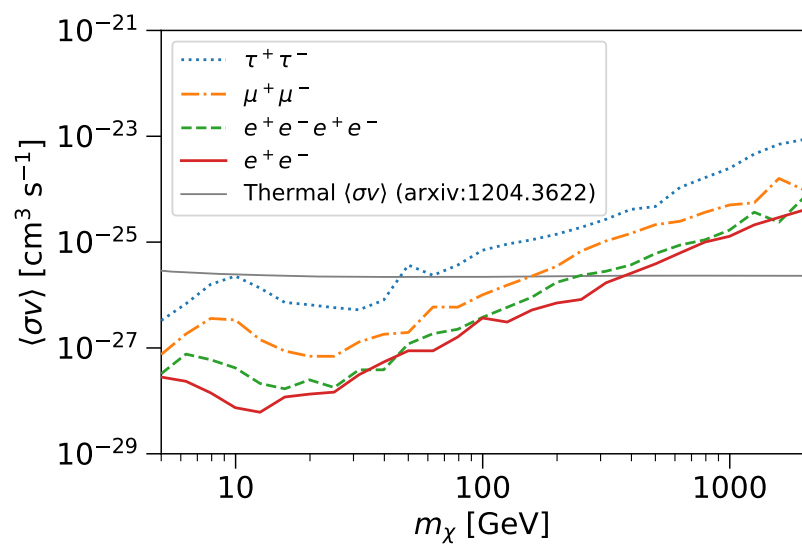

Figure 3: Dark matter limits for different leptophilic final states for a dark matter mass range from 5 to $2000 \mathrm{GeV}$ for decays into $\tau^{+} \tau^{-}$(blue), $\mu^{+} \mu^{-}$(orange), $e^{+} e^{-} e^{+} e^{-}$(green, dashed) and $e^{+} e^{-}$(red), and the thermal cross section (gray) from [24]. Our limits rule out a thermal annihilation cross section for dark matter masses below between 60 to $300 \mathrm{GeV}$ depending on the final state. order of $\sim 2.5 \cdot 10^{-28} \mathrm{~cm}^{3} / \mathrm{s}$, allowing us to more generically rule out models with even $\sim 1 \%$ annihilation to $e^{+} e^{-}$final states. Notably, these constraints also have implications for the constraints on the hard-to reach muonic channels discussed in [26]. Finally, our results improve on the existing limits by Bergström et al. [7] by a factor of $\sim 2$, and extend the limits to higher dark matter masses.

As the dark matter contribution is proportional to the halo height, we repeat this analysis with a halo height of $3 \mathrm{kpc}$ as a lower limit on the halo height [25], and obtain similar results, see [22].

\section{References}

[1] Webpage. https://ams02.space/.

[2] D. Hooper, P. Blasi, and P. D. Serpico, JCAP 01, 025 (2009), arXiv:0810.1527 [astro-ph] .

[3] S. Profumo, Central Eur. J. Phys. 10, 1 (2011), arXiv:0812.4457 [astro-ph] .

[4] T. Linden and S. Profumo, Astrophys. J. 772, 18 (2013), arXiv:1304.1791 [astro-ph.HE] .

[5] H. Yuksel, et al., Phys. Rev. Lett. 103, 051101 (2009), arXiv:0810.2784 [astro-ph] .

[6] D. Malyshev, et al., Phys. Rev. D 80, 063005 (2009), arXiv:0903.1310 [astro-ph.HE] .

[7] L. Bergstrom, T. Bringmann, I. Cholis, D. Hooper, and C. Weniger, Phys. Rev. Lett. 111, 171101 (2013), arXiv:1306.3983 [astro-ph.HE] .

[8] M. Aguilar, L. Ali Cavasonza, G. Ambrosi, L. Arruda, N. Attig, P. Azzarello, A. Bachlechner, F. Barao, and et. al. (AMS Collaboration), Phys. Rev. Lett. 122, 041102 (2019). 
[9] M. Aguilar, L. Ali Cavasonza, B. Alpat, G. Ambrosi, L. Arruda, N. Attig, S. Aupetit, P. Azzarello, and et. al. (AMS Collaboration), Phys. Rev. Lett. 121, 051101 (2018).

[10] M. Aguilar, D. Aisa, B. Alpat, A. Alvino, G. Ambrosi, K. Andeen, L. Arruda, N. Attig, and et. al. (AMS Collaboration), Phys. Rev. Lett. 114, 171103 (2015).

[11] M. Aguilar, D. Aisa, B. Alpat, A. Alvino, G. Ambrosi, K. Andeen, L. Arruda, N. Attig, and et. al. (AMS Collaboration), Phys. Rev. Lett. 115, 211101 (2015).

[12] "Galprop," https://galprop.stanford.edu/.

[13] I. Cholis, D. Hooper, and T. Linden, (2020), arXiv:2007.00669 [astro-ph.HE] .

[14] L. Gleeson and W. Axford, Astrophys. J. 154, 1011 (1968).

[15] D. R. Lorimer, IAU Symp. 218, 105 (2004), arXiv:astro-ph/0308501 .

[16] D. Lorimer et al., Mon. Not. Roy. Astron. Soc. 372, 777 (2006), arXiv:astro-ph/0607640 .

[17] J. F. Navarro, C. S. Frenk, and S. D. M. White, Astrophys. J. 462, 563 (1996), arXiv:astro$\mathrm{ph} / 9508025$.

[18] P. Gondolo, J. Edsjo, P. Ullio, L. Bergstrom, M. Schelke, and E. A. Baltz, JCAP 07, 008 (2004), arXiv:astro-ph/0406204 .

[19] J.-F. Fortin, J. Shelton, S. Thomas, and Y. Zhao, (2009), arXiv:0908.2258 [hep-ph] .

[20] https://johannesbuchner.github.io/PyMultiNest/.

[21] https://iminuit.readthedocs.io/en/stable/.

[22] I. John and T. Linden, (2021), arXiv:2107.10261 [astro-ph.HE] .

[23] F. Feroz and M. P. Hobson, Mon. Not. Roy. Astron. Soc. 384, 449 (2008), arXiv:0704.3704 [astro-ph] .

[24] G. Steigman, B. Dasgupta, and J. F. Beacom, Phys. Rev. D 86, 023506 (2012), arXiv:1204.3622 [hep-ph] .

[25] M. Korsmeier and A. Cuoco, (2021), arXiv:2103.09824 [astro-ph.HE] .

[26] R. K. Leane, T. R. Slatyer, J. F. Beacom, and K. C. Y. Ng, Phys. Rev. D 98, 023016 (2018), arXiv:1805.10305 [hep-ph] .

[27] R. Trotta, G. Jóhannesson, I. V. Moskalenko, T. A. Porter, R. R. d. Austri, and A. W. Strong, Astrophys. J. 729, 106 (2011), arXiv:1011.0037 [astro-ph.HE] .

[28] G. Jóhannesson et al., Astrophys. J. 824, 16 (2016), arXiv:1602.02243 [astro-ph.HE] .

[29] I. Cholis, D. Hooper, and T. Linden, Phys. Rev. D 95, 123007 (2017), arXiv:1701.04406 [astro-ph.HE] . 